\title{
EDITORIAL
}

\section{If good things come from above, do bad things come from below?}

\author{
David M Findlay* \\ See related research by Tanamas et al., http://arthritis-research.com/content/12/2/R58
}

\begin{abstract}
Factors in the synovial fluid that maintain healthy articular cartilage, such as hyaluronic acid and lubricin, come from above. Is it possible that factors which lead to the destruction of cartilage come from below in the subchondral bone? The recent acquisition of tools to probe early events in osteoarthritis is shedding new light on possible contributions from this compartment on the initiation and progression of the disease. Tanamas and co-workers now provide evidence that bone marrow lesions in the subchondral bone are predictive, both of loss of cartilage and of formation of subchondral cysts. These data provoke questions about the nature and role of bone marrow lesions.
\end{abstract}

Finding the factors that initiate, or the mechanisms that lead to progression of, osteoarthritis (OA) has proven frustrating and largely unproductive. Identification of risk factors for the condition - such as prior trauma to the joint, elevated body weight and female sex - may have helped with management of OA but has done little to progress understanding of the underlying factors that drive it. OA research has been more difficult than research for some other diseases of the skeleton, for several important reasons. Early OA, at the level of symptoms, can be episodic, making it difficult to identify the disease and to follow it longitudinally. Since the main early symptom is pain, clinical trials of new therapies have been problematic. Animal experiments have been bedevilled by a lack of models that accurately replicate the human disease. And perhaps, as argued by a minority of workers in the field, disease initiators have been sought in the wrong place; that is, cartilage versus bone.

*Correspondence: david.findlay@adelaide.edu.au

Discipline of Orthopaedics and Trauma, University of Adelaide, Level 4 Bice Building, Royal Adelaide Hospital, Adelaide 5000, South Australia, Australia
The recent study of Tanamas and colleagues highlights the way in which new-generation imaging holds the promise of shedding new light on this old problem [1]. In particular, high-resolution magnetic resonance imaging (MRI) can now deliver objective, measurable information about all structures of the joint, including the amount and quality of articular cartilage, and is also a powerful tool to investigate the subchondral bone. The holy grail of clinical investigation, namely longitudinal study with quantitative endpoints, is now accessible for OA. What Tanamas and colleagues' study shows is important because it adds to emerging evidence that processes in the subchondral bone relate strongly to changes in the volumetric amount of articular cartilage. Specifically, bone marrow lesions (BMLs), the mysterious MRI-bright regions in the subchondral bone that occur more commonly in OA, were shown to be predictive of loss of cartilage and of formation of subchondral cysts. In turn, cysts were more likely than BMLs to occur in association with loss of cartilage.

These data pose the intriguing question of whether BMLs encode key clues to the aetiology of OA. Longitudinal studies have shown that the presence of BMLs constitutes a potent risk factor for structural deterioration in knee OA [2]. BML enlargement has been strongly associated with increased cartilage loss, and Tanamas and colleagues' data further suggest that their conversion into cysts is even more predictive of cartilage loss. Significantly, a reduction in the extent of BMLs on MRI has been shown to associate with a decrease in cartilage degradation [3]. Since the origin of BMLs is not known, its investigation needs to be prioritised as an important research topic. Current informed guesses are that BMLs comprise regions of oedema, perhaps secondary to episodes of local ischaemia. Although it is not possible to biopsy BMLs in patients with early OA, several studies have sought to correlate the MRI findings with histology in more severe disease. Regions of BMLs in end-stage OA patients at knee replacement were more likely to exhibit oedema, bone necrosis and trabecular abnormalities than were control sites [4]. 
If BMLs are secondary to local ischaemia in the subchondral bone, there are several possible consequences. Firstly, the supply of nutrients and oxygen from regions of ischaemic subchondral bone, to the overlying articular cartilage, might be reduced. Cartilage nutrition has been considered to derive from the synovial fluid. The work of Imhof and colleagues, however, suggested that more than $50 \%$ of the glucose, oxygen and water requirements of cartilage are provided by perfusion from the subchondral vessels [5]. They described the dense subchondral vasculature in close proximity to the cartilage, and the micro-channels that penetrate the subchondral mineralisation zone and permit communication between the bone and the cartilage. More recent work indicates that small molecules can diffuse, in healthy joints, bidirectionally from the synovial compartment into the cartilage and underlying bone and from the subchondral bone into the overlying cartilage [6]. Inspection of the osteochondral junction of long bones reveals that osteocytes and osteocyte canaliculi, which are also probable conduits of nutrients, are intimately associated with the articular cartilage. Experimental interruption of contact between articular cartilage and subchondral bone results in degeneration of the cartilage, and osteoblasts from OA subchondral bone conferred catabolic changes in articular chondrocytes [7].

Secondly, osteocyte death in bone is becoming recognised as a signalling event for osteoclastic removal of the nonviable bone and its replacement in a remodelling episode [8]. Although subchondral bone is constantly being remodelled, concentration of this activity in a particular region of the bone could alter its mechanical integrity and its ability to properly support the overlying cartilage.

Tanamas and colleagues conclude that cysts (and BMLs) may provide therapeutic targets for the treatment of knee OA [1]. Certainly, the recent acquisition of tools to probe early events in subchondral bone in OA should deliver rapid advances in our understanding of the natural history of this condition.

\section{Abbreviations}

$\mathrm{BML}$, bone marrow lesion; $\mathrm{MRI}$, magnetic resonance imaging; $\mathrm{OA}$ osteoarthritis.

\section{Competing interests}

The author declares that he has no competing interests.

Published: 27 May 2010

\section{References}

1. Tanamas SK, Wluka AE, Pelletier JP, Martel-Pelletier J, Abram F, Wang Y, Cicuttini FM: The association between subchondral bone cysts and tibial cartilage volume and risk of joint replacement in people with knee osteoarthritis: a longitudinal study. Arthritis Res Ther 2010, 12:R58.

2. Felson DT, McLaughlin S, Goggins J, LaValley MP, Gale ME, Totterman S, Li W, Hill C, Gale D: Bone marrow edema and its relation to progression of knee osteoarthritis. Ann Intern Med 2003, 139:330-336.

3. Garnero P, Peterfy C, Zaim S, Schoenharting M: Bone marrow abnormalities on magnetic resonance imaging are associated with type II collagen degradation in knee osteoarthritis: a three-month longitudinal study. Arthritis Rheum 2005, 52:2822-2829.

4. Zanetti M, Bruder E, Romero J, Hodler J: Bone marrow edema pattern in osteoarthritic knees: correlation between MR imaging and histologic findings. Radiology 2000, 215:835-840.

5. Imhof H, Sulzbacher I, Grampp S, Czerny C, Youssefzadeh S, Kainberger F: Subchondral bone and cartilage disease: a rediscovered functional unit. Invest Radio/ 2000, 35:581-588.

6. Pan J, Zhou X, Li W, Novotny JE, Doty SB, Wang L: In situ measurement of transport between subchondral bone and articular cartilage. $J$ Orthop Res 2009, 27:1347-1352.

7. Sanchez C, Deberg MA, Piccardi N, Msika P, Reginster JY, Henrotin YE: Subchondral bone osteoblasts induce phenotypic changes in human osteoarthritic chondrocytes. Osteoarthritis Cartilage 2005, 13:988-997.

8. Noble B: Bone microdamage and cell apoptosis. Eur Cell Mater 2003, 6:46-55

doi:10.1186/ar3007

Cite this article as: Findlay DM: If good things come from above, do bad things come from below? Arthritis Research \& Therapy 2010, 12:119. 